\title{
Machine-Learning-Aided Optical OFDM for Intensity Modulated Direct Detection
}

\author{
Xiaoyu Zhang, Member, IEEE, Thien Van Luong, Periklis Petropoulos, Fellow, OSA, \\ and Lajos Hanzo, Fellow, IEEE
}

\begin{abstract}
End-to-end learning systems are conceived for Orthogonal Frequency Division Multiplexing (OFDM)-aided optical Intensity Modulation paired with Direct Detection (IM/DD) communications relying on the Autoencoder (AE) architecture in deep learning. We first propose an AE-aided Layered ACO-OFDM (LACO-OFDM) scheme, termed as LACONet, for exploiting the increased bandwidth efficiency of LACO-OFDM. LACONet employs a Neural Network (NN) at the transmitter for bit-tosymbol mapping, and another $\mathrm{NN}$ at the receiver for recovering the data bits, which together form an $\mathrm{AE}$ and can be trained in an end-to-end manner for simultaneously minimizing both the BER and PAPR. Moreover, the detection architecture of LACONet is drastically simplified compared to classical LACO-OFDM, since the Fast Fourier Transform (FFT) operation is applied only once at the receiver. We further propose a generalized AE-aided optical OFDM scheme for IM/DD communications, termed as IMDD-OFDMNet, where the unipolarity of the Time Domain (TD) signal is no longer guaranteed by the Hermitian Symmetry, but rather by taking the absolute square value of the complex TD signal. As such, all available subcarriers of IMDDOFDMNet are used for carrying useful information, hence it has a higher throughput than the LACO-based schemes. As a further benefit, its transceiver requires only a single Inverse FFT or FFT. Finally, simulation results are provided to show that our learning schemes achieve better BER and PAPR performance than their conventional counterparts.
\end{abstract}

Index Terms-Optical communications, autoencoder, OFDM, LACO-OFDM, optical OFDM, bit error ratio, peak-to-averagepower ratio, neural network, deep learning, visible light communications.

\section{INTRODUCTION}

There is an insatiable appetite for improving the data rates to accommodate flawless multimedia services [1]. As a result, an increasing amount of attention has been dedicated to

The authors would like to acknowledge the financial support of the Engineering and Physical Sciences Research Council (EPSRC) projects EP/P034284/1 and EP/P003990/1 (COALESCE). L. Hanzo also acknowledges financial support through the European Research Council's Advanced Fellow Grant QuantCom (Grant No. 789028).

Xiaoyu Zhang is with the School of Electronics and Computer Science, University of Southampton, Southampton, SO17 1BJ, U.K., and also with the Optoelectronics Research Centre, University of Southampton, Southampton, SO17 1BJ, U.K. (e-mail: xy.zhang@soton.ac.uk).

Thien Van Luong was the School of Electronics and Computer Science, University of Southampton, Southampton, SO17 1BJ, U.K. He is now with the Faculty of Computer Science, Phenikaa University, Hanoi 12116 , Vietnam, and also with the Phenikaa Research and Technology Institute (PRATI), A\&A Green Phoenix Group JSC, Hanoi 11313, Vietnam. (e-mail: thien.luongvan@phenikaa-uni.edu.vn).

Periklis Petropoulos is with the Optoelectronics Research Centre, University of Southampton, Southampton, SO17 1BJ, U.K. (e-mail: pp@orc.soton.ac.uk).

Lajos Hanzo is with the School of Electronics and Computer Science, University of Southampton, Southampton, SO17 1BJ, U.K. (e-mail: lh@ecs.soton.ac.uk).
Optical Wireless Communications (OWC) for exploiting its rich bandwidth resources in the unlicensed optical spectrum [2].

As opposed to coherent detection techniques, Intensity Modulation paired with Direct Detection (IM/DD) has gained popularity in OWC as a benefit of its inexpensive hardware implementation [3]. In the simplest IM/DD setup, a Light Emitting Diode (LED) operating at a visible wavelength acts as the Transmitter (TX), converting the information bits into optical intensity and transmitting them. At the Receiver (RX) side, a PhotoDiode (PD) is capable of converting the received optical signal back to the electric domain for further signal processing. This appealingly simple design makes it economical to widely roll out OWC. However, one of the major drawbacks of IM/DD is that the transmitted electric signal driving the optical emitter has to be positive and realvalued.

This unipolar real-valued constraint prevents OWC from adopting the Orthogonal Frequency Division Multiplexing (OFDM) scheme to create Optical OFDM (O-OFDM) [2], [4], [5]. This impediment has been partially circumvented by arranging for the Frequency Domain (FD) signal to satisfy the so-called Hermitian Symmetry (HS), which guarantees for its Time Domain (TD) counterpart to be real-valued. Armstrong [6] then proposed the Asymmetrically Clipped Optical OFDM (ACO-OFDM) scheme, which achieves clipping all the negative-valued TD samples without information loss by inserting zeros into every even-indexed FD subcarriers. However, ACO-OFDM achieves this at the cost of low Bandwidth Efficiency (BE), since three quarters of the total bandwidth conveys zeros [7]. Harnessing the multi-layer modulation concept [8], Wang et al. [9] later proposed the Layered ACOOFDM (LACO-OFDM) scheme, which arranges for the blank subcarriers of ACO-OFDM to be beneficially exploited, hence improving the $\mathrm{BE}$, albeit at the cost of an increased complexity, as discussed in [10]-[12]. LACO-OFDM has also been shown to reduce the Peak-to-Average Power Ratio (PAPR) [10] and improve flexibility [13], [14].

Recent years have seen an increasing trend of harnessing Machine Learning (ML) or Deep Learning (DL) in the physical layer of communication systems [15]-[17], including the specific tasks of classification [18], coding [19], channel estimation and signal detection [20]-[22], scheduling [23], [24], just to name a few. On a broader note, ML has also been exploited for holistic system optimization based on the appealing concept of the so-called Autoencoder (AE) [16]. Explicitly, in an AE scheme, NNs are used instead of the 
classic signal processing modules at both TX and RX sides. At the TX side, the original information is directly mapped by an $\mathrm{AE}$ to the transmitted signal, which is then sent through the communication channel. The NN decoder located at the RX is capable of detecting the received signal and reproducing a close replica of the transmitted bit sequence with the aid of unsupervised training [15]. This is different from supervised training, where the training data are mapped to labels beforehand and the NN learns the mapping strategy used during the training process. Explicitly, the DNN at the transmitter and the DNN at the receiver constitute a single network. The training process ensures that the input to the transmitter-side of the DNN and the output of the receiverside of the DNN are as similar as possible, through the use of the mean squared error (MSE) loss in the training, without accounting for their labels.

In contrast to the classic Shannonian "block-based" communication systems, the AE-based scheme constitutes a "combined" design, which is capable of jointly optimizing the entire signal processing chain of a communication system without having to consider each Shannonian block separately. Hence, AEs have found their way into a wide range of communications scenarios, such as Radio Frequency (RF) wireless [15], optical fiber [25] and optical wireless systems [26]. Explicitly, in [27], Felix et al. embedded the AE scheme into an OFDM system relying on the conventional Cyclic Prefix (CP) for amalgamating the advantages of both learning and OFDM. In [19], Cammerer et al. exploited the adoption of channel coding to the AE system with experimental validation in RF. In [28], Luong et al. proposed the novel concept of AE for noncoherent multi-carrier systems. As a further advance, Karanov et al. [25], [29] studied the AE in the context of optical fiber communications and provided experimental results to prove its performance benefits. Further optical physical layer performance investigations have been carried out by Hao et al. [30] and Ulkar et al. [31] in the context of ACO-OFDM and other scenarios, respectively.

It is worth mentioning that in our work the key OFDM components, namely the IFFT and FFT layers at the transmitter and receiver side respectively, are not replaced by the neural networks. Despite the fact that given a sufficiently long training stage, the neural networks can eventually "learn" to use OFDM, it has been demonstrated in [15], [27] that explicitly introducing the IFFT and FFT layers provides additional expert knowledge for the neural network, which expedites training and improves the performance. Moreover, using the IFFT/FFT processing ensures that the system benefits from the compelling advantages of OFDM, such as multi-carrier modulation and single-tap equalization. The practice of artificially injecting expert knowledge is not uncommon in machine learning. One of the most popular examples is the utilization of convolutional neural networks (CNN) in computer vision [16], where manually introducing convolutional layers speeds up the training and reduces the complexity.

Against this background, we propose a pair of novel AEaided O-OFDM schemes, termed as LACONet based on LACO-OFDM and the generalized IMDD-OFDMNet. We contrast our contributions to the relevant state-of-the-art at a glance in Tab. I, which are detailed below:

1) Proposal of LACONet: We propose an AE-aided LACO-OFDM scheme for Visible Light Communications (VLC) and fiber optic communications, where the FD symbols to be loaded onto each LACO-OFDM layer are determined by the $\mathrm{NN}$-based $\mathrm{AE}$, rather than on the classic Quadrature Amplitude Modulation (QAM) mappers. This allows us to simultaneously improve both the Bit Error Ratio (BER) and PAPR performance of conventional LACO-OFDM. At the receiver, the NN decoder recovers all the data bits using a single Fast Fourier Transform (FFT) operation, which drastically reduces the complexity of conventional LACO-OFDM, where the number of FFTs to be used is determined by that of the layers.

2) Proposal of IMDD-OFDMNet: Further, we propose a generalized AE-aided optical OFDM scheme, where the unipolarity of the TX TD signal no longer has to be guaranteed by the HS. More explicitly, in contrast to both our LACONet and to conventional LACO-OFDM, all subcarriers of IMDD-OFDMNet are used for carrying useful information, which results in a higher BE than the LACO-based schemes despite its lower TX complexity, since it requires only a single Inverse FFT (IFFT) at the transmitter. By contrast, the number of IFFT activation in conventional LACO-OFDM is given by the number of layers included in the scheme. Despite its simplified TX architecture, IMDD-OFDMNet achieves better BER and PAPR than both LACONet and classic LACO-OFDM.

This treatise is organized as follows: Section II introduces the IM/DD channel we are using, as well as the LACO-OFDM scheme designed for IM/DD transmission. Our LACONet is presented in Section III, followed by IMDD-OFDMNet in Section IV. Finally, our conclusions are offered in Section V.

\section{SySTEM MODELS}

\section{A. Intensity Modulated Direct Detection Optical Channel}

In the IM/DD transmitter, the information is mapped onto the current driving an optical emitter, such as an LED for visible light communications or a laser for free space optics and fiber optics, which controls its instantaneous illuminating intensity. As long as the driving current lies within the linear range of the optical emitter, the illuminating intensity is linearly proportional to it. Hence, after transmission through the optical channel, the beam impinging on the photodiode at the RX side is then converted back into the electric domain further processing. Naturally, mapping the information onto the intensity of light requires the current exciting the optical transmitter to be positive-valued.

Similar to RF signals, IM/DD transmission may also suffer from Frequency-Selective (FS) fading, due to the potential existence of both Line-Of-Sight (LOS) and Non-LOS (NLOS) light beams impinging at the photodiode of the RX [32]. Therefore, it is natural to appropriately adapt and transplant mature anti-fading techniques of classic RF communications into IM/DD solutions. 
TABLE I

A COMPARISON OF RESEARCH WORKS ON LEARNING-AIDED OPTICAL WIRELESS COMMUNICATIONS

\begin{tabular}{|l|c|c|c|c|c|c|}
\hline Topic & [29] & [32] & [19] & [30] & [31] & This work \\
\hline \hline AE for end-to-end communications & $\checkmark$ & $\checkmark$ & $\checkmark$ & $\checkmark$ & $\checkmark$ & $\checkmark$ \\
\hline Multi-objective optimization & $\checkmark$ & $\checkmark$ & $\checkmark$ & $\checkmark$ & & $\checkmark$ \\
\hline Application to optical communications & $\checkmark$ & $\checkmark$ & & $\checkmark$ & $\checkmark$ & $\checkmark$ \\
\hline Application to OFDM & & & $\checkmark$ & $\checkmark$ & & $\checkmark$ \\
\hline Proposal of an HS-based O-OFDM scheme & & & $\checkmark$ & & & $\checkmark$ \\
\hline Proposal of another non-HS-based O-OFDM scheme & & & & & & $\checkmark$ \\
\hline
\end{tabular}

Explicitly, for FS fading, we consider the scenario of the popular indoor environment of [33], where an LED transmitter and a PD receiver are in an empty room of dimension $(5.0 \mathrm{~m} \times$ $5.0 \mathrm{~m} \times 3.0 \mathrm{~m})$. The LED is placed at the center of the ceiling at the coordinate of $(2.5 \mathrm{~m}, 2.5 \mathrm{~m}, 3.0 \mathrm{~m})$, while the PD is located in the corner of the room at coordinate $(0.5 \mathrm{~m}, 1.0 \mathrm{~m}, 0.0 \mathrm{~m})$ pointing upwards. Moreover, we consider the coexistence of both a line-of-sight path and two non-line-of-sight paths in the channel, whose linear gain and time delays are $(3.8,1.4,0.5) \times$ $10^{-5}$ and $(11,14,17) \mathrm{ns}$, respectively.

\section{B. Optical OFDM based on Hermitian Symmetry}

In RF-OFDM, the information bits are usually mapped to complex-valued QAM symbols, before baseband modulation using IFFT modules for transforming them from the FD to the TD, yielding a TD modulated signal that is generally also complex-valued, which is unsuitable for driving the optical emitter in IM/DD transmission, as discussed above. However, by carefully arranging the FD sequence to satisfy Hermitian symmetry, it would be guaranteed that the corresponding TD signal is indeed positive and real-valued. Explicitly, a length$K$ FD sequence $s$ satisfying Hermitian symmetry exhibits an anti-symmetric FD spectrum, which means that its second half is represented by the conjugated counterpart of its first half in reversed order, which is formulated as:

$$
s[k]=-s^{*}[K-k-1], \quad 0 \leq k \leq \frac{K}{2}-1,
$$

where ()$^{*}$ represents the complex conjugate operation. Next, we will briefly outline both ACO-OFDM and LACO-OFDM.

1) ACO-OFDM: Fig. 1 provides the schematic of the ACOOFDM transmitter and receiver, which obeys Hermitian symmetry. At the transmitter, the ACO-OFDM scheme leaves all the even-indexed subcarriers blank, so that when the negative samples of the TD signal are clipped to zero, all the clipping distortions directly fall on those blank subcarriers as detailed in [10], leaving the information-bearing odd-indexed subcarriers free from distortion. Correspondingly, at the receiver, the PD converts the received optical signal back to the electric signal $\boldsymbol{r}$, while the FFT operation converts $\boldsymbol{r}$ back to the FD signal $\overline{\boldsymbol{R}}$. The odd-indexed subcarriers from the first half of $\overline{\boldsymbol{R}}$ can then be extracted as $\boldsymbol{R}$ for obtaining the bit sequence $\hat{b}$ through QAM demapping.

Again, a major drawback of ACO-OFDM is, however, its low BE. According to its bit-to-subcarrier mapping strategy, only a quarter of all subcarriers can be utilized for carrying "payload" information, while the rest are either used for accommodating Hermitian symmetry or the clipping distortion. Under the framework of Hermitian symmetry, various sophisticated O-OFDM schemes [9], [34]-[37] have been proposed for improving the BE by exploiting the "clippingdistortion-bearing" subcarriers, which occupy half of the total bandwidth.

2) LACO-OFDM: Fig. 2 provides the schematic of the LACO-OFDM system, where additional layers are overlaid onto the ACO-OFDM signal for loading extra information on the clipping-distortion-bearing subcarriers. Explicitly, if the original ACO-OFDM (referred to as layer 1 signal) utilizes a $K$-point IFFT, layer 2 would have an ACO-OFDM signal using a $K / 2$-point IFFT, corresponding to the $K / 2$ idle subcarriers in layer 1. Likewise, layer 3 would employ ACOOFDM relying on $K / 4$-point IFFT and layer $l$ with $K / 2^{l-1}$ point IFFT. Therefore, more information can be conveyed by the additional layers using the same bandwidth, which improves the BE. As a further benefit, it has also been shown in [10] that having more layers reduces the PAPR of LACOOFDM.

However, while the preparation of each layer can be independently and simultaneously carried out, the detection process shown in the bottom half of Fig. 2 is relatively complex, because the signals of the higher layers are mapped to the same subcarriers as the clipping distortions of all the previous layers. For example, the subcarriers conveying the layer 2 signal also accommodate the clipping distortion of layer 1, while the subcarriers of layer 3 additionally contain the clipping distortion of layer 1 and layer 2. Hence, in contrast to the parallel transmitter structure of Fig. 2, the detection of LACOOFDM is carried out on a layer-by-layer basis. Explicitly, after converting the received TD signal $r$ into the FD, the subcarriers conveying the message of layer 1 are extracted and detected. The resultant bits are then re-modulated with the objective of re-generating the corresponding clipping distortion, which will be subtracted from the originally received signal for decontaminating it from the interference imposed by layer 1 , paving the way for the detection of layer 2 . The extracted and detected symbols of layer 2 will also have to be re-modulated for detecting layer 3. This detection loop of Fig. 2 keeps on operating until all layers become detected, during which its issue of high-complexity is observed.

Explicitly, the detection process of a single layer includes the FFT, layer-extraction, symbol-to-bit demapping, reloading, IFFT and clipping, as seen in Fig. 2. In contrast to the parallel architecture of the transmitter, the layers must be sequentially detected at the receiver. Hence, the improved BE 


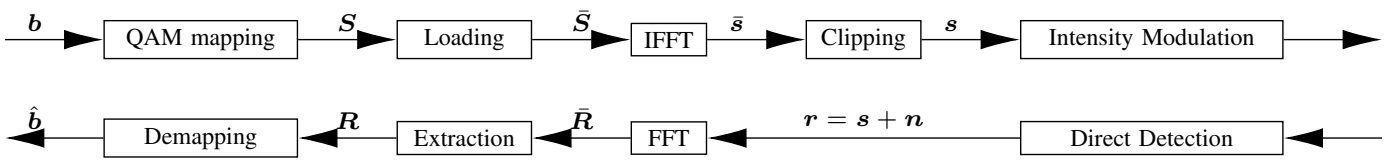

Fig. 1. Schematic of the ACO-OFDM transmitter and receiver.

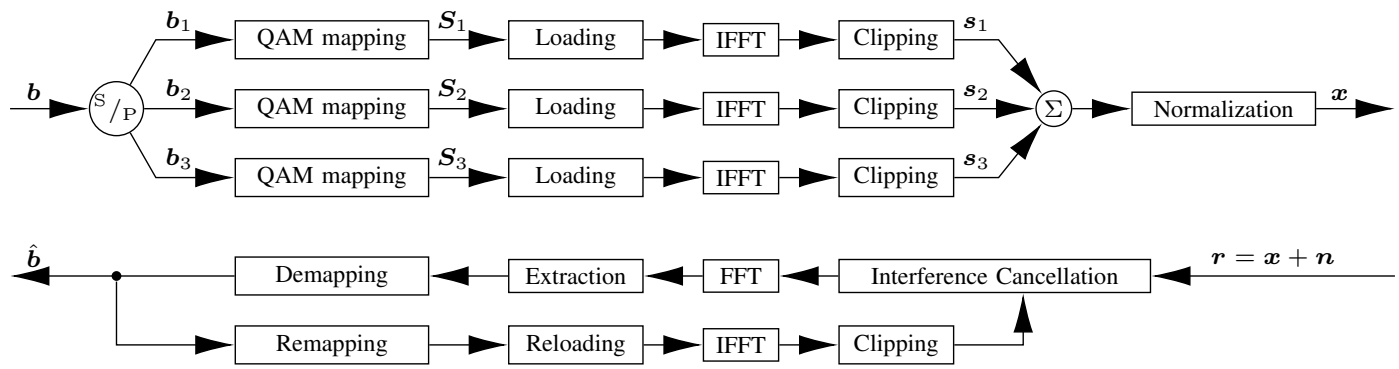

Fig. 2. Transmitter and receiver schematic of LACO-OFDM.

is attained at the cost of an increased receiver complexity and signal processing time delay. To tackle this issue, we resort to harnessing artificial intelligence, where an $\mathrm{AE}$ is employed at the transmitter and receiver, yielding our LACONet.

\section{LACONET}

In this section, we present our novel LACONet scheme. To avoid ambiguity, we have to carefully distinguish a LACO layer of transmitting signal from an NN layer.

\section{A. LACONet Transceiver Design}

Fig. 3 shows the schematic of the proposed LACONet transceiver, where the upper half is the transmitter, while the lower half is the receiver. Meanwhile, the detailed structures of the NN-Based AE $f(\cdot)$ and the NN-Based Detector $g(\cdot)$ of Fig. 3 are illustrated in Fig. 4.

The LACONet transmitter of Fig. 3 is similar to the original LACO-OFDM transmitter of Fig. 2. Both designs feature a layered ACO-OFDM-style process and rely on layer superposition. However, while the bit stream of LACO-OFDM is mapped to symbols by QAM modules, an NN-based encoder in employed by LACONet for bit-to-symbol mapping. Let us represent the NN-base encoding process by $f(\cdot)$ and the encoded symbols of Fig. 3 by $\boldsymbol{Z}$, yielding:

$$
\boldsymbol{Z}=f(\boldsymbol{b})=f(\boldsymbol{X}),
$$

where $\boldsymbol{X}$ is the symbol-based counterpart of the bit sequence $\boldsymbol{b}$, having $X[k]=b[k]$ with $k=1, \ldots, K$ representing the bit-index in a symbol.

The symbols $Z$ are then mapped to the layers of Fig. 3 for further layered processing through a serial-to-parallel $(\mathrm{S} / \mathrm{P})$ operation, where in Fig. 3 we have $L=3$ :

$$
\boldsymbol{Z}=\left[\boldsymbol{Z}_{1}, \boldsymbol{Z}_{2}, \ldots, \boldsymbol{Z}_{L}\right]
$$

where $Z_{l}$, being of length $K / 2^{l+1}$, represents the symbol stream mapped to LACO layer $l$. The NN of AE handles the mapping of bits/symbols to the LACO layers, since the NN output $\boldsymbol{Z}$ of Fig. 3 is sorted for the layers. The symbols of each LACO layer are then parsed through the ACO-OFDM modules, including the IFFT-based modulation and clipping, which are collectively represented by:

$$
\boldsymbol{U}_{l}=\mathcal{F}^{-1}\left(\boldsymbol{Z}_{l}\right) \text {. }
$$

The outputs $\boldsymbol{U}_{l}(l=1, \ldots, L)$ of each LACO layers' clipper are then superimposed, which is formulated as:

$$
\boldsymbol{U}_{\Sigma}=\sum_{l=1}^{L} \boldsymbol{U}_{l}
$$

followed by normalization representing the power control [28], given as:

$$
\boldsymbol{U}=\frac{\sqrt{E_{\mathrm{s}}} \boldsymbol{U}_{\Sigma}}{\sqrt{\mathbb{E}\left[\left\|\boldsymbol{U}_{\Sigma}\right\|^{2}\right]}},
$$

where the operation $\mathbb{E}\left[\|\cdot\|^{2}\right]$ in the denominator calculates the statistical average power of the output signal, and the factor $\sqrt{E_{\mathrm{s}}}$ in the numerator ensures that the average output power is $E_{\mathrm{s}}$. Upon transmitting the signal $\boldsymbol{U}$ through the optical channel, the received signal becomes:

$$
\boldsymbol{Y}=\boldsymbol{h} \otimes \boldsymbol{U}+\boldsymbol{n},
$$

where $\boldsymbol{n}$ denotes the Gaussian-distributed noise and $\boldsymbol{h}$ is the channel impulse response, while $\otimes$ is the convolution operation. When an FS fading channel is considered, we have $\boldsymbol{h}=\left[h_{0}, h_{1}, \ldots, h_{I-1}\right]$ representing the gain from of the $I$ paths, while for when an AWGN channel, we have simply $\boldsymbol{h}=[1]$.

Recall that the benefit of using an AE-aided design becomes more explicit at the receiver side, which in this case simply has two components. The received signal is converted to the FD by the FFT-based demodulator $\mathcal{F}(\cdot)$, which is then directly entered into the NN-based detector $g(\cdot)$. Therefore, the receiver process can be formulated as:

$$
\hat{\boldsymbol{X}}=(g \circ \mathcal{F})(\boldsymbol{Y}),
$$

where "o" represents the function composition operator. The NN-based detector is trained so that the signal on all LACO 


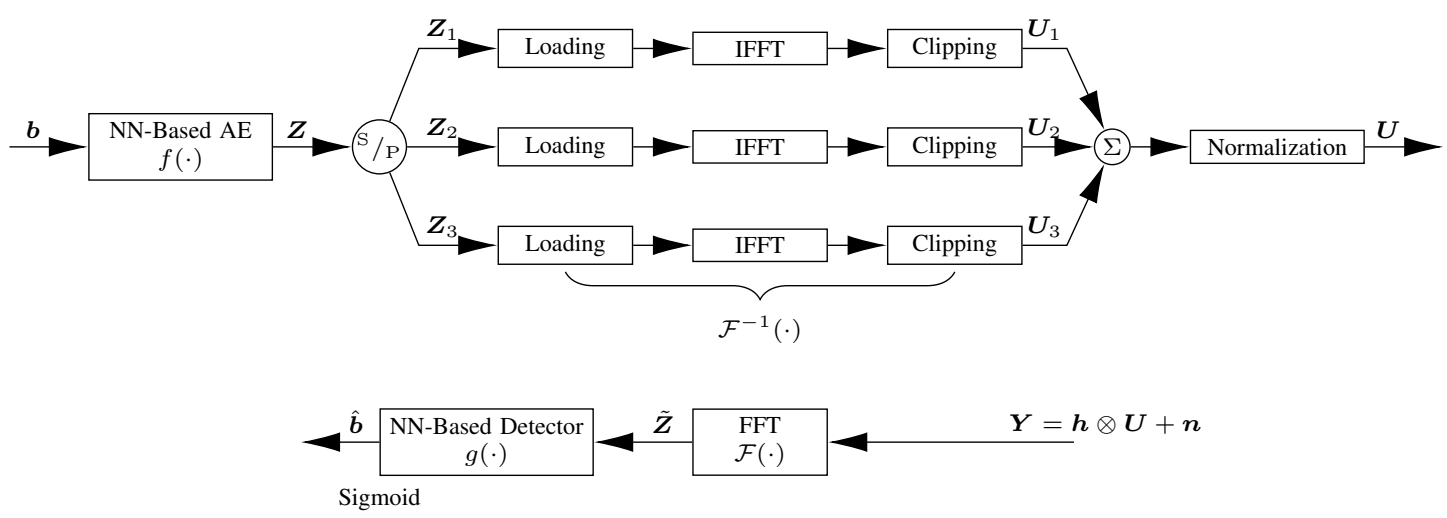

Fig. 3. Transmitter and receiver schematic of LACONet.
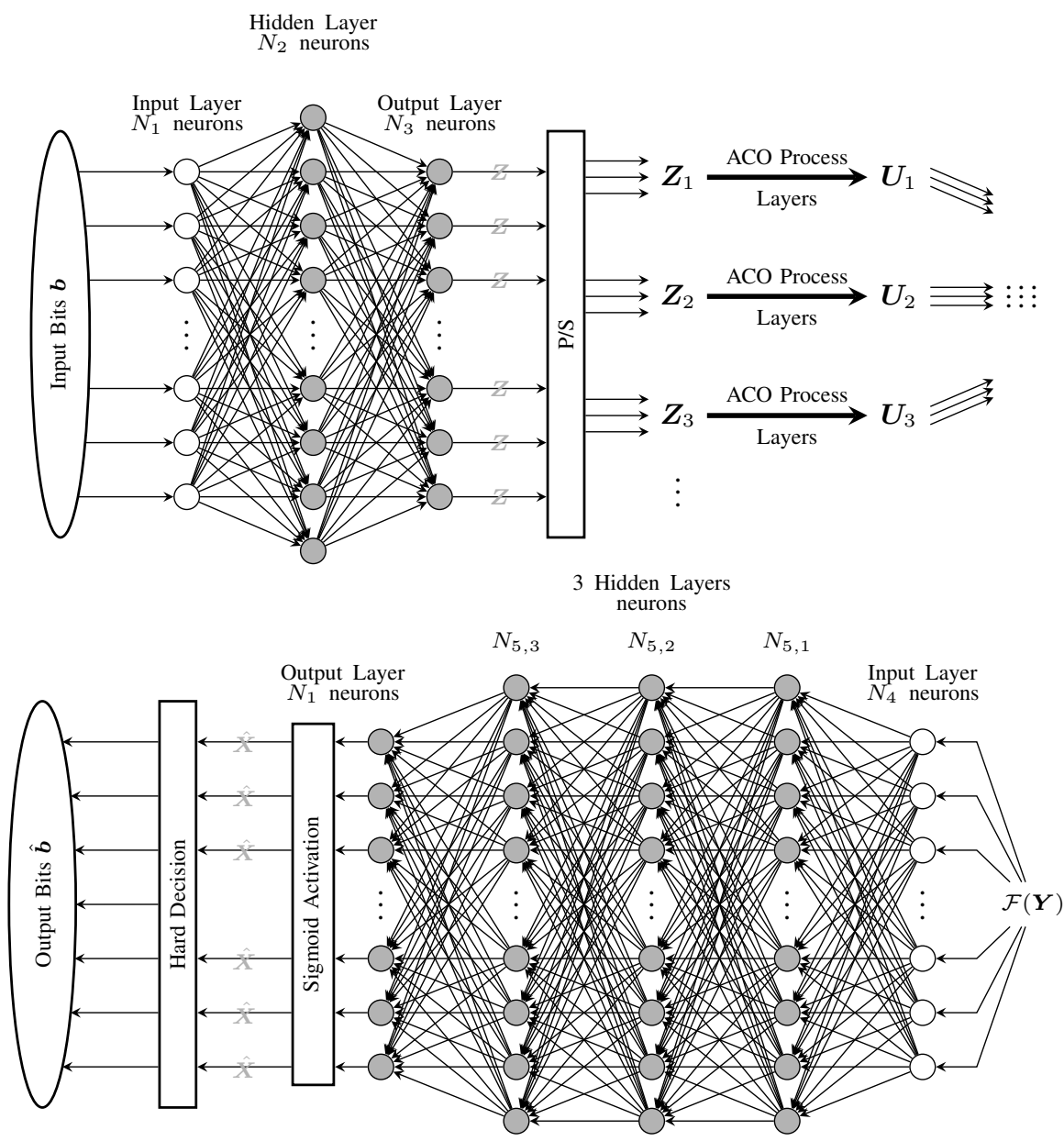

Fig. 4. Illustration of the neural networks in the proposed LACONet. 
layers can be simultaneously detected. The bit stream $\hat{b}$ of Fig. 3 can then be directly obtained after a hard decision operation at the receiver's output as:

$$
\hat{b}[k]=\left\{\begin{array}{l}
1, \text { if } \hat{X}[k]>0.5, \\
0, \text { otherwise, }
\end{array}\right.
$$

where the Sigmoid activation function of [22] is used for the output layer of the RX NN, whose values, i.e. the elements of $\hat{\boldsymbol{X}}$, are in the range of $(0,1)$.

\section{B. Neural Network Training}

By employing our proposed LACONet, we aim at improving both the BER and PAPR, relative to a LACO-OFDM signal with the same number of LACO layers and BE. Therefore, a pair of loss functions are required for representing the BER and PAPR behavior.

Explicitly, for minimizing the BER, a loss function $\mathcal{L}_{\mathrm{BER}}$ can be defined as the mean square distance between the transmitted symbols $\boldsymbol{X}$ and their estimate $\hat{\boldsymbol{X}}$ :

$$
\mathcal{L}_{\mathrm{BER}}(\boldsymbol{X}, \hat{\boldsymbol{X}})=\frac{1}{N_{1}} \sum_{n=1}^{N_{1}}|X(n)-\hat{X}(n)|^{2},
$$

where $N_{1}$ is the number of bits per transmission frame and $|\cdot|$ is the absolute value operation.

By contrast, the loss function representing the PAPR is defined as:

$$
\mathcal{L}_{\mathrm{PAPR}}(\boldsymbol{U})=\frac{\max _{k}|U(k)|^{2}}{\frac{1}{K} \sum_{k=1}^{K}|U(k)|^{2}}-1,
$$

where $K$ is the number of subcarriers, the numerator $\max _{k}|U(k)|^{2}$ stands for the peak sample energy in signal $\boldsymbol{U}$, whereas the denominator $\frac{1}{K} \sum_{k=1}^{K}|U(k)|^{2}$ represents its average sample energy. It is worth noting that $\mathcal{L}_{\mathrm{PAPR}}(\boldsymbol{U})$ is differentiable, since each component of $|U(k)|^{2}$ in the maximization operation is differentiable. Meanwhile, it is clear that the fraction in the first term has a minimum value of 1 , when the signal has a constant envelope. Therefore, the term " -1 " has been included for ensuring that the PAPR-loss tends to 0 , similarly to the BER-loss.

The overall loss function $\mathcal{L}$ used for the $\mathrm{NN}$ training has to consider both. Hence, a loss scaling factor $\alpha$ is introduced to represent the specific weight of PAPR-loss, yielding

$$
\mathcal{L}=\mathcal{L}_{\mathrm{BER}}+\alpha \mathcal{L}_{\mathrm{PAPR}}
$$

Let us now introduce a two-stage training procedure, where the first stage is focused on minimizing the PAPR, while the second stage on the BER. Explicitly,

1) In the first stage (initial training), a relatively high scaling factor $\alpha$ is employed, so that the PAPR performance has a stronger influence on the optimization process. A higher learning rate $r_{\text {learning }}$ is used for accelerating the training process.

2) In the second stage (retraining), a smaller value of $\alpha$ is stipulated, so that the learning becomes biased towards improving the BER performance, without unduly eroding the PAPR reduction attained. Correspondingly, $r_{\text {learning }}$ is also reduced for fine-tuning the system. The values adopted for $\alpha$ and $r_{\text {learning }}$ are detailed in the next section.

\section{LACONet Performance and Discussions}

Let us now study the performance of LACONet by comparing its BER for different numbers of LACO layers, BE, training complexity, as well as against the original LACOOFDM. Unless otherwise specified, the parameters of Tab. II are adopted for all simulations in this section. Observe from Tab. II that we employ the batch normalization technique of [38] after each hidden layer of both the TX and RX NNs to accelerate the training by reducing the internal covariate shift ${ }^{1}$, while the Rectified Linear Unit (ReLU) activation of [22] is used for all of their hidden layers. Additionally, it should be noted that we consciously use linear activation for the output layer of the TX NN, so that no restriction is imposed on the FD symbols $Z$ of Fig. 3.

Fig. 5 shows our BER comparison between LACONet and LACO-OFDM for both AWGN and FS fading channels, where different BEs are characterized. All other parameters, including the number of NN layers and number of neurons within each NN layer, are summarized in Tab. II. Explicitly, the BEs of $0.75,1.5$ and $0.875 \mathrm{bps} / \mathrm{Hz}$ are considered. Hence, the performance of conventional LACO-OFDM schemes having 2-layer 4QAM, 2-layer 4QAM and 2-layer 16QAM are characterized in Fig. 5 for benchmarking the performance of LACONet. The associated BEs are calculated as in Tab. III, where the BEs of layers 1,2 and 3 are $1 / 4$, $1 / 8$ and $1 / 16$ that of the bits per info-bearing subcarrier, respectively, as elaborated in Sec. II-B2. Observe in Fig. 5 that the BER of LACONet is better than that of LACO-OFDM for all three BEs. It also transpires that the Signal-to-Noise Ratio (SNR) improvement of the 3-layer 4QAM scheme is more significant than that of the 2-layer 4QAM and 16QAM schemes for all BEs. This indicates that LACONet succeeds in more beneficially reducing the inter-layer interference than LACO-OFDM in the face of having more layers. Moreover, Fig. 5 b shows a modest performance degradation under the FS channel of Tab. II due to the multi-path effects for the 2-layer 16QAM scenario of $1.5 \mathrm{bps} / \mathrm{Hz}$ BE. Nevertheless, the OFDMbased channel equalization of both LACONet and LACOOFDM succeeds in eliminating most of the FS channel-effects. Moreover, observe again by comparing Fig. $5 \mathrm{~b}$ to Fig. 5a that the gaps between LACONet and LACO-OFDM of signals at same BE become wider for FS channels, which indicates that LACONet is more capable of combating the multipath effects than classic OFDM. This is because the transmitter and receiver of LACONet are jointly optimized during the training stage in the presence of the FS channel, in order to achieve the best performance under practical channel conditions. On the other hand, the O-OFDM transceiver designs without AI assistance are not optimized for any specific channel.

\footnotetext{
${ }^{1}$ The internal covariate shift is the change of the statistical distribution of each layer's parameters during the training process, as the result of the change of its previous layers. [38]
} 
TABLE II

Default Parameters Used for Simulations in Section III

\begin{tabular}{|c|c|c|}
\hline Parameter name & Value & $\begin{array}{c}\text { Applicable } \\
\text { Figures }\end{array}$ \\
\hline \multicolumn{3}{|l|}{ Parameters for all schemes simulated } \\
\hline Number of subcarriers $K$ & 32 & $5,8,9$ \\
\hline Number of LACO layers $L$ & 2 & $6,8,9$ \\
\hline Number of mapped symbols on LACO layer $1 K_{1}=K / 4$ & 8 & 8,9 \\
\hline Number of mapped symbols on LACO layer $2 K_{2}=K / 8$ & 4 & 8,9 \\
\hline \multicolumn{3}{|l|}{ Optical channel } \\
\hline AWGN channel impulse response $\boldsymbol{h}$ & {$[1]$} & $\begin{array}{r}5 \mathrm{a}, 6,8 \mathrm{a}, 9 \\
10,11,12\end{array}$ \\
\hline FS channel impulse response $\boldsymbol{h}$ & {$[3.8,1.4,0.5]$} & $5 b, 8 b$ \\
\hline \multicolumn{3}{|l|}{ Parameters for LACONet } \\
\hline Bandwidth efficiency $q / K$ & 1.5 & $6,8,9$ \\
\hline Total number of information bits $q$ & 48 & 8,9 \\
\hline \multicolumn{3}{|l|}{ TX NN Input Layer } \\
\hline Number of neurons $N_{1}=q$ & 48 & 8,9 \\
\hline TX Hidden Layer: Number of Layers & 1 & $5,6,8,9$ \\
\hline Number of neurons $N_{2}$ & 128 & $5,6,8,9$ \\
\hline Batch normalization after each hidden layer & Yes & $5,6,8,9$ \\
\hline Layer Activation & $\mathrm{ReLu}$ & $5,6,8,9$ \\
\hline \multicolumn{3}{|l|}{ TX Output Layer } \\
\hline Number of neurons $N_{3}=2\left(K_{1}+K_{2}\right)$ & 24 & $5,6,8,9$ \\
\hline Layer Activation & Linear & $5,6,8,9$ \\
\hline \multicolumn{3}{|l|}{ RX NN Input Layer } \\
\hline Number of neurons $N_{4}=K$ & 32 & $5,8,9$ \\
\hline RX Hidden Layer: Number of Layers & 3 & $5,6,8,9$ \\
\hline Number of neurons $N_{5}=\left[N_{5,1}, N_{5,2}, N_{5,3}\right]$ & {$[512,256,128]$} & $5,6,8,9$ \\
\hline Batch normalization after each hidden layer & Yes & $5,6,8,9$ \\
\hline Layer Activation & $\mathrm{ReLu}$ & $5,6,8,9$ \\
\hline \multicolumn{3}{|l|}{ RX Output Layer } \\
\hline Number of neurons $N_{1}$ & 48 & 8,9 \\
\hline Layer Activation & Sigmoid & $5,6,8,9$ \\
\hline \multicolumn{3}{|l|}{ Initial training } \\
\hline PAPR loss scale $\alpha$ & 0.005 & $5,6,8,9$ \\
\hline Learning rate $r_{\text {learning }}$ & 0.001 & $5,6,8,9$ \\
\hline Number of training epochs $n_{\text {ini }}$ & 2000 & $5,6,8,9$ \\
\hline \multicolumn{3}{|l|}{ Retraining } \\
\hline PAPR loss scale $\alpha$ & 0.0001 & $5,6,8,9$ \\
\hline Learning rate $r_{\text {learning }}$ & 0.0001 & $5,6,8,9$ \\
\hline Number of training epochs $n_{\text {re }}$ & 200 & $5,6,8,9$ \\
\hline \multicolumn{3}{|l|}{ Parameters for LACO-OFDM } \\
\hline QAM constellation size for all 2 LACO layers $M$ & 16 & $6,8,9$ \\
\hline Bandwidth efficiency $(1 / 4+1 / 8) \log _{2} M$ & 1.5 & $6,8,9$ \\
\hline
\end{tabular}

TABLE III

LACO-OFDM BANDWIDTH EFFICIENCYS (BPS/Hz) CALCULATION

\begin{tabular}{l|ccc}
\hline Case & 1 & 2 & 3 \\
\hline $\begin{array}{l}\text { Number of layers } \\
\text { QAM Order }\end{array}$ & 2 & 3 & 2 \\
Bits per info- & 2 & 4 & 2 \\
bearing subcarrier & & & \\
layer 1 BE & 0.5 & 0.5 & 1 \\
layer 2 BE & 0.25 & 0.25 & 0.5 \\
layer 3 BE & N/A & 0.125 & N/A \\
Overall BE & 0.75 & 0.875 & 1.5 \\
\hline
\end{tabular}

Fig. 6 shows the Complementary Cumulative Distribution Functions (CCDF) of the PAPR of both LACONet and LACOOFDM having two LACO layers and different numbers of subcarriers $K$. The number of input bits $N_{1}$ was adjusted to $1.5 \mathrm{~K}$ for each scheme to align their BEs for a fair comparison, while all other parameters are summarized in Tab. II. Observe in Fig. 6 that LACONet exhibits a lower PAPR than its LACO-OFDM counterpart for all three cases.
By contrast, no significant difference can be observed amongst the three LACONet schemes' PAPR performance. Note from this investigation that the hidden layer specifications of all three LACONet scenarios were adjusted to meet their specific throughput target. Explicitly, for the 16-subcarrier LACONet, we have $N_{2}=128$ and $\boldsymbol{N}_{5}=[256,256,64]$, while $N_{2}=512$ and $\boldsymbol{N}_{5}=[512,512,512]$ are used for the 64-subcarrier scheme.

We can conclude from this section that both the BER and PAPR performance of LACO-OFDM can be significantly improved with the aid of NN, despite its simplified detector architecture, since several IFFT-based re-modulation and iterative interference cancellation stages of LACO-OFDM were dispensed with, as seen in Fig. 3. However, given the requirement Hermitian symmetry, half of the bandwidth still has to be sacrificed in order to carry conjugate symbols. This limitation will be circumvented in the next section. 


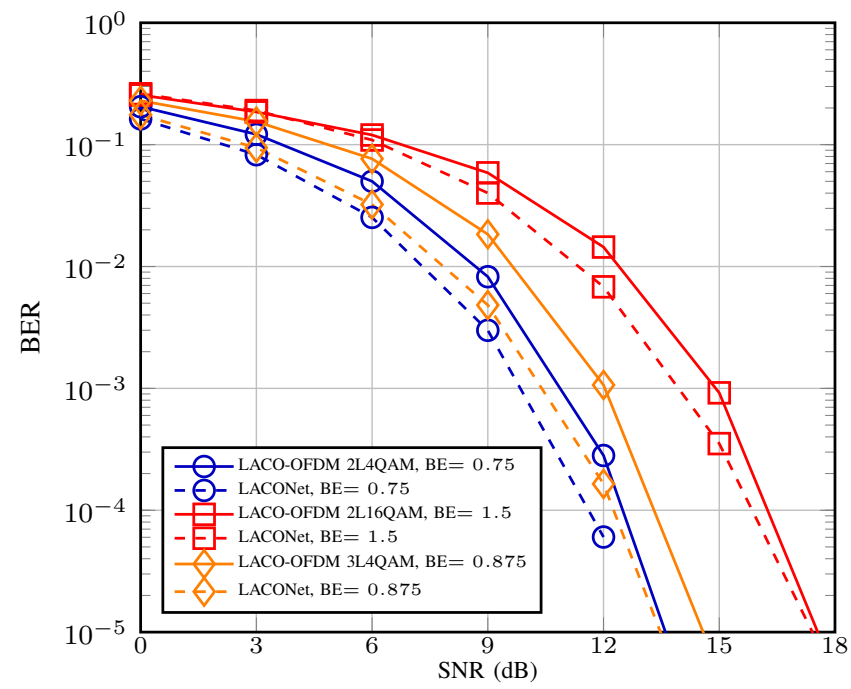

(a) AWGN Channel

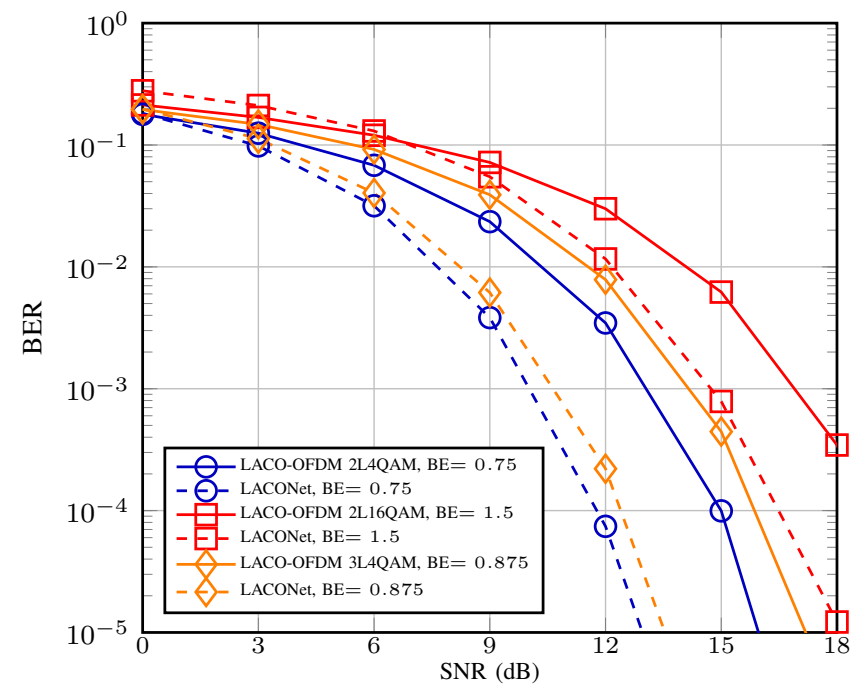

(b) FS Channel

Fig. 5. BER versus SNR for LACONet for bandwidth efficiencies of 0.75 , 1.5 and $0.875 \mathrm{bps} / \mathrm{Hz}$ attained by varying the number of neurons $N_{1}$ of the TX input NN layer and RX output NN layer. All other simulation parameters are summarized in Tab. II.

\section{IMDD-OFDMNET}

Inspired by the performance vs. complexity improvement of LACONet, we proceed by demonstrating the NNs are also capable of directly generating unipolar signals under the OFDM framework.

\section{A. IMDD-OFDMNet Architecture}

Fig. 7 shows the schematic of the IMDD-OFDMNet transceiver. While the AE-aided LACONet of Fig. 3 still relies on LACO-OFDM-like components at the transmitter, the IMDD-OFDMNet scheme of Fig. 7 provides increased freedom for the NN training by eliminating the Hermitian Mapping blocks of Fig. 3. Thus, while a LACONet system having $K$ subcarriers and $L$ LACO layers produces a sequence of $K\left(1-2^{-L}\right)$ symbols at the output layer of the $\mathrm{NN}$ based TX, the IMDD-OFDMNet system of Fig. 7 succeeds in

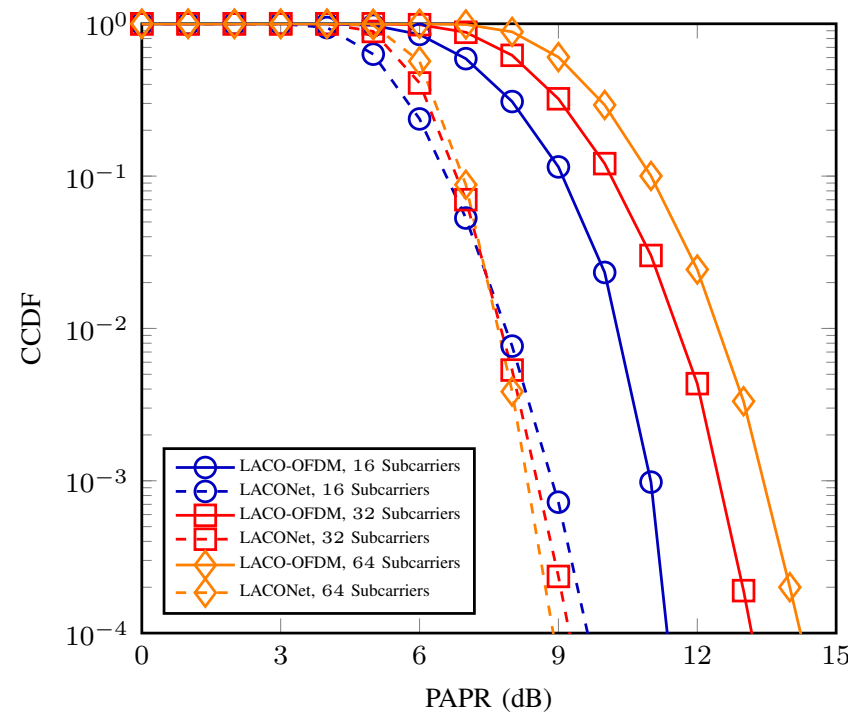

Fig. 6. CCDF of the signal PAPR for LACONet with different number of OFDM subcarriers.

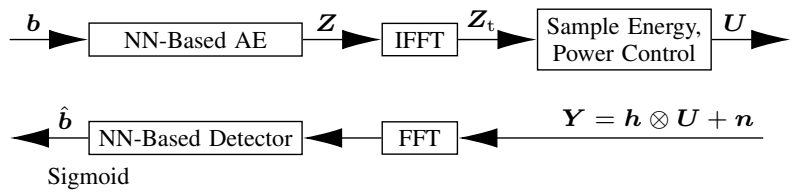

Fig. 7. Transmitter and receiver schematic of IMDD-OFDMNet.

further increasing this to $K$, because no Hermitian Mapping is required.

Akin to LACONet, the bit stream $\boldsymbol{b}$ is entered into the AE of Fig. 7, which outputs a symbol sequence $Z$ of the same length as the number of subcarriers $K$. In the absence of a carefully designed Hermitian Mapper, the TD signal $Z_{\mathrm{t}}$ of Fig. 7 obtained by the IFFT-based modulation now becomes bi-polar and complex-valued. To create a unipolar signal, the energy of each TD sample of $\boldsymbol{Z}_{\mathrm{t}}$ is calculated for constructing the signal $\boldsymbol{U}$ as follows:

$$
U[k]=\min \left(Z_{\mathrm{t}}[k] \cdot Z_{\mathrm{t}}^{*}[k], U_{\max }\right), k=0,1, \ldots, K-1,
$$

where $\min \left(\cdot, U_{\max }\right)$ is the clipping operation that limits the maximum amplitude of the signal to $U_{\max }$. Following the power control operation similar to that of (6), the resultant signal is then used for intensity modulation.

Moreover, the FFT-NN-based receiver of Fig. 3 designed for LACONet is retained for the IMDD-OFDMNet receiver.

For training the IMDD-OFDMNet scheme of Fig. 7, a similar approach to that of Section III-B can be adopted. Given a total of $K$ subcarriers available, to achieve a BE of $q / K$, an input bit sequence $\mathbf{b}$ of length $q$ is required. Again, both loss functions of (10), (11) and (12), as well as the two-stage training process of Section III-B, are employed for jointly minimizing the BER and PAPR.

\section{B. IMDD-OFDMNet Performance and Discussions}

In this section, we present numerical results for characterizing the performance of our proposed IMDD-OFDMNet, where 
TABLE IV

Default Parameters Used FOR Simulations in SECtion IV

\begin{tabular}{lr}
\hline \multicolumn{1}{c}{ Parameter name } & Value \\
\hline Parameters for IMDD-OFDMNet & AWGN \\
Optical channel & 32 \\
Number of subcarriers $K$ & 1.5 \\
Bandwidth efficiency $q / K$ & 48 \\
Total number of information bits $q$ & 48 \\
Number of neurons on the TX NN input layer $N_{1}=q$ & 48 \\
Number of neurons on the RX NN output layer $N_{1}=q$ (Sigmoid activation) & 1 \\
Number of TX AE NN hidden layers & 128 \\
Number of neurons on the TX NN hidden layers $N_{2}$ & Yes \\
Batch normalization after each hidden layer & 64 \\
Number of neurons on the TX NN output layer $N_{3}=2 K$ (Linear activation) & 32 \\
Number of neurons on the RX NN input layer $N_{4}=K$ & 3 \\
Number of RX NN Hidden Layers (ReLU activation) & $328]$ \\
Number of neurons on the RX NN hidden layers $N_{5}=\left[N_{5,1}, N_{5,2}, N_{5,3}\right]$ & Yes \\
Batch normalization [39] after each hidden layer & \\
Initial training & 0.005 \\
PAPR loss scale $\alpha$ & 2000 \\
Number of training epochs & \\
Retraining & 0.0005 \\
PAPR loss scale $\alpha$ & 200 \\
Number of training epochs &
\end{tabular}

the default parameters are summarized in Tab. IV. By contrast, when referring to the benchmarks of LACONet and LACOOFDM, the parameters of Tab. II are used.

1) BER and PAPR: Fig. 8 shows the BER comparison of IMDD-OFDMNet, LACONet and LACO-OFDM under both AWGN and FS fading channels, where the BE of all schemes is $1.5 \mathrm{bps} / \mathrm{Hz}$. For LACONet and LACO-OFDM, two LACO layers are used. Additionally, for LACO-OFDM, 16QAM is adopted for both LACO layers. According to Fig. 8, despite its reduced TX complexity, IMDD-OFDMNet outperforms LACONet for the same NN setup. Furthermore, observe in Fig. 8b an approximately $3 \mathrm{~dB} E_{b} / N_{0}$ for IMDD-OFDMNet over LACO-OFDM at a BER of $10^{-5}$.

Moreover, we present the BER and PAPR performance of both LACONet and IMDD-OFDMNet, when trained under a finite clipping threshold, by the dashed lines in Figs. 9, respectively. A $10 \mathrm{~dB}$ clipping ratio, corresponding to $U_{\max }=$ $\sqrt{1 \mathrm{~W} \times 10 \cdot \log _{10}\left(10^{10 / 10}\right)} \approx 3.16 \mathrm{~V}$ has been used in (13). It is clear that for LACONet, a slight PAPR improvement is achieved after training under the finite clipping, albeit at the cost of degraded BER performance, especially at higher SNRs $\left(E_{b} / N_{0}>12 \mathrm{~dB}\right)$, where the clipping distortion might be the dominant source of error. On the other hand, IMDDOFDMNet has a slightly better PAPR reduction than LACONet, at a negligible BER penalty. This also demonstrates the better performance of IMDD-OFDMNet thanks to its higher AI optimization freedom. However, it is worth noting that LACONet still outperforms LACO-OFDM under clipping. Although the former also suffers from clipping distortion, yet it does not have any PAPR gain.

To elaborate further, Fig. 9 shows the CCDF of the PAPR of IMDD-OFDMNet, LACONet and LACO-OFDM, with all parameters specified in Tabs. II and IV. Explicitly, IMDDOFDMNet provides even further PAPR reduction compared to LACONet.

2) Offline Learning Complexity: In contrast to classical methods, the improved performance of learning-aided schemes is closely related to the quality of offline training. In Fig. 10, we plot the BER of IMDD-OFDMNet as a function of the number of training epochs, which represents the associated offline complexity. It is clear that the system only starts to perform adequately after at least 100 epochs, when the BER dips below the $10^{-3}$ threshold, which is a typical value for which forward error correction becomes capable of cleaning up the residual errors [39]. Additionally, the BER gradually approaches that of perfect training after about 500 epochs, after which no more significant BER improvement can be observed. Furthermore, Fig. 10 also validates the efficiency of our proposed 2-stage training strategy, as detailed in Section III-B and Tab. IV. While the first 500 epochs of initial training result in considerable BER reduction, the improvements slow down after 1000 epochs. On the other hand, by changing the cost function and learning rate in the retraining stage according to Tab. IV, an additional $50 \sim 200$ epochs result in a $3 \mathrm{~dB}$ SNR gain at $\mathrm{BER}=10^{-4}$, as the dashed lines shown in Fig. 10 .

The trend can be viewed more clearly in Fig. 11, where the BER curve is the $E_{b} / N_{0}$ distance for reaching the $10^{-3} \mathrm{BER}$ compared to the minimum $E_{b} / N_{0}$ observed, while similarly the PAPR curve is the PAPR reduction for reaching the $10^{-3}$ PAPR CCDF compared to the minimum PAPR observed. A $0.3 \mathrm{~dB}$ PAPR penalty is observed after retraining at 2000 epochs, which is the price paid in exchange for a $0.3 \mathrm{~dB}$ $E_{b} / N_{0}$ improvement observed at BER $=10^{-3}$.

3) Online Processing Complexity: In this section, we analyze the online signal processing complexities of both the 


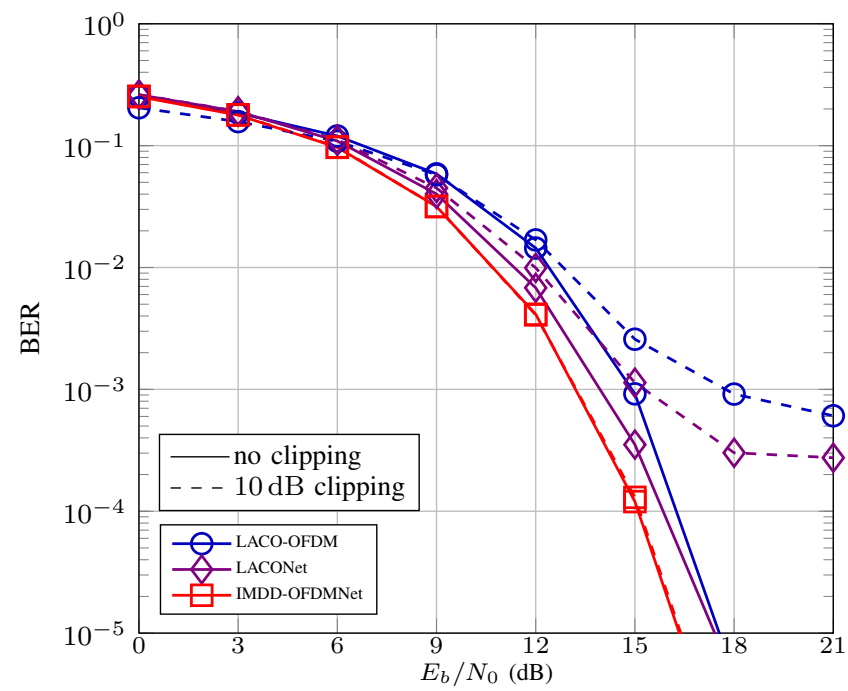

(a) AWGN Channel

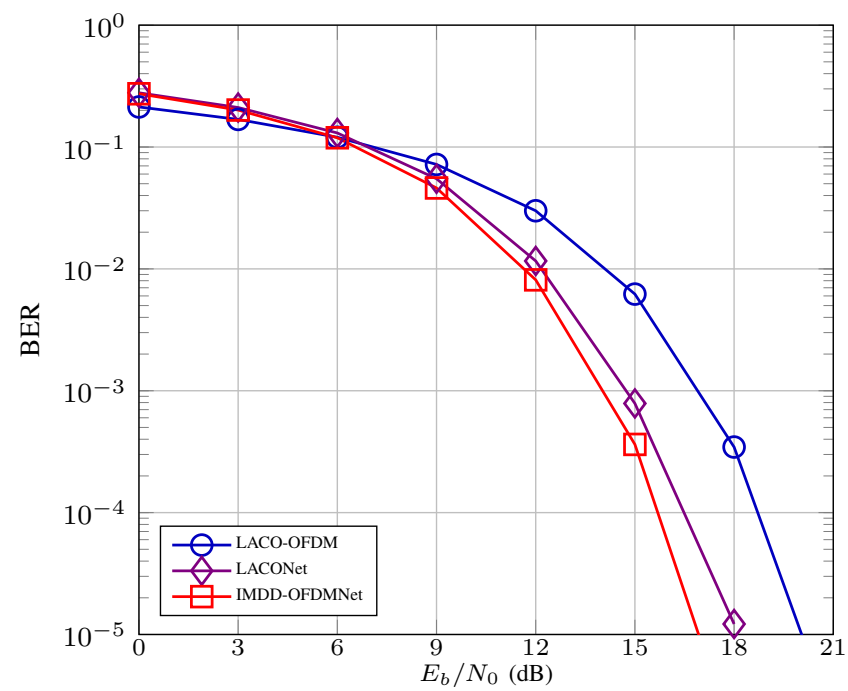

(b) FS Channel

Fig. 8. BER versus SNR for IMDD-OFDMNet, LACONet and LACOOFDM schemes, all having a bandwidth efficiency of $1.5 \mathrm{bps} / \mathrm{Hz}$, using the parameters of Tab. II for LACO-OFDM and LACONet and of Tab. IV for IMDD-OFDMNet. Dashed lines represent signal trained under $10 \mathrm{~dB}$ clipping and solid lines the absent.

proposed LACONet and IMDD-OFDMNet in terms of the number of Real-valued Multiplications and Additions (RMAs) involved and compare them to that of conventional ACO- and LACO-OFDM operating without any learning. By inspecting the schematics seen in Figs. 3 and 7, it becomes plausible that both the IFFT/FFT and the AE NNs impose the highest signal processing complexity. However, recall that the situations are different at the TX and RX side, hence have to be separately discussed. For simplifying the analysis, we disregard the OFDM CP overhead and additional complexity reduction of ACO-OFDM attained by omitting the processing of the blank subcarriers, because these two assumptions apply to all schemes involved and therefore the comparisons remain valid.

A $K$-point FFT/IFFT operation requires $K \log _{2} K$ complex additions and $\frac{K}{2} \log _{2} K$ complex multiplications, which are equivalent to $3 K \log _{2} K$ real additions and $2 K \log _{2} K$ real

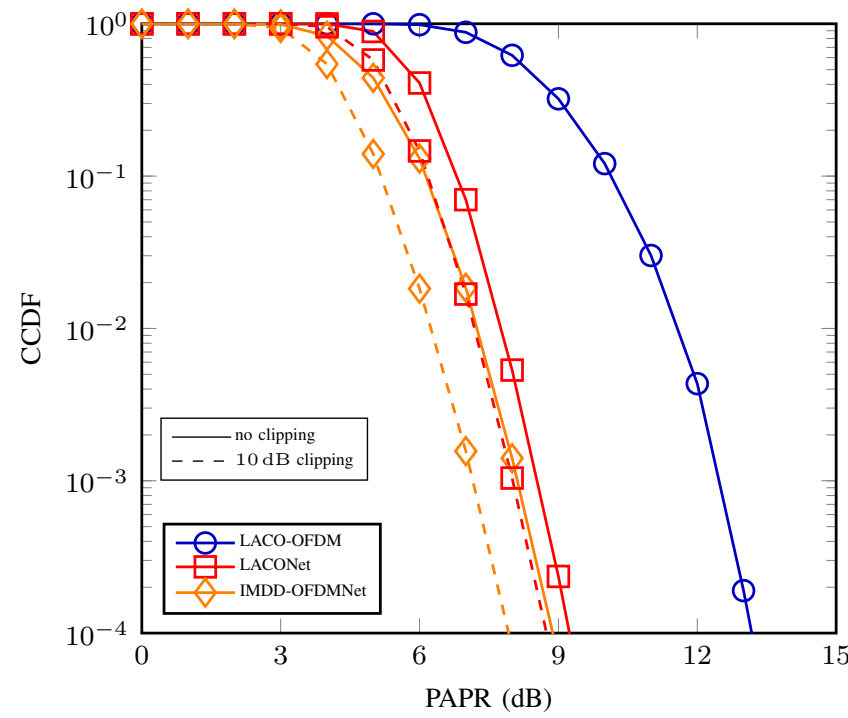

Fig. 9. CCDF of PAPR for IMDD-OFDMNet, LACONet and LACO-OFDM schemes having 32 subcarriers. Dashed lines represent signal trained under $10 \mathrm{~dB}$ clipping and solid lines the absent.

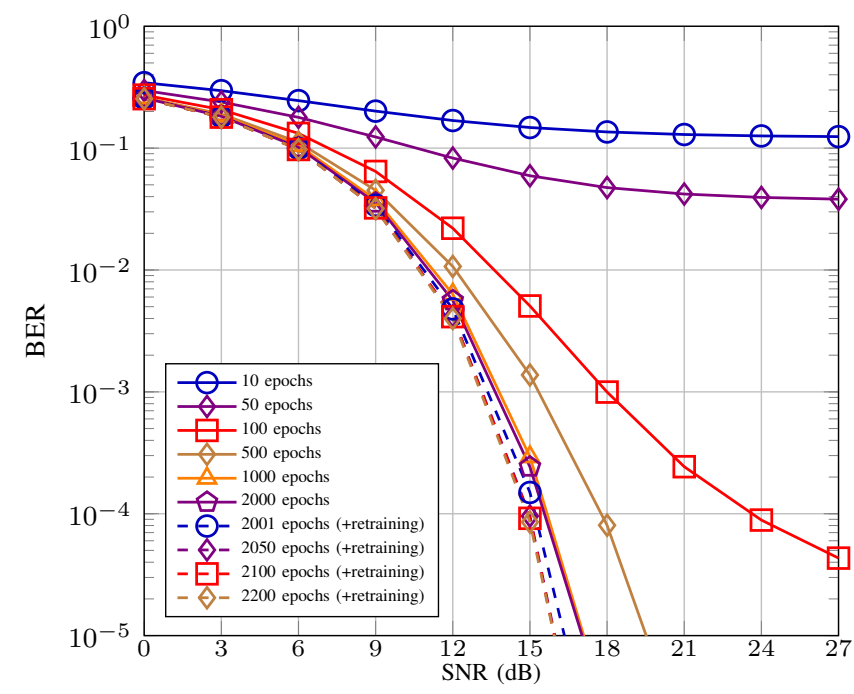

Fig. 10. BER versus SNR for IMDD-OFDMNet with different number of training epochs.

multiplications, leading to a total of $C_{1}=5 K \log _{2} K$ RMAs. Hence, for ACO-OFDM having $K$ subcarriers, both its TX and RX require $C_{1}$ RMAs for IFFT/FFT, respectively. This also applies to IMDD-OFDMNet, because it requires only a single IFFT/FFT. By contrast, for LACO-OFDM having $L$ layers, the TX complexity is given by the sum of processing all LACO layers, where each layer has half the complexity of its predecessor due to its halved number of symbols. As a result, the LACO-OFDM TX requires $\sum_{l=1}^{L}\left(\frac{1}{2^{L-1}} C_{1}\right)=\left(2-2^{1-L}\right) C_{1}$ RMAs. This also applies to LACONet, due to its similar TX structure. On the other hand, the LACO-OFDM RX imposes nearly twice the complexity of ACO-OFDM, which is due to the extra requirement of reconstructing the LACO-layer's signal after detection. Explicitly, the LACO-OFDM RX requires $\sum_{l=1}^{L}\left(\frac{1}{2^{L-1}} C_{1}\right)+\sum_{l=1}^{L-1}\left(\frac{1}{2^{L-1}} C_{1}\right)=\left(4-2^{1-L}-2^{2-L}\right) C_{1}$ 


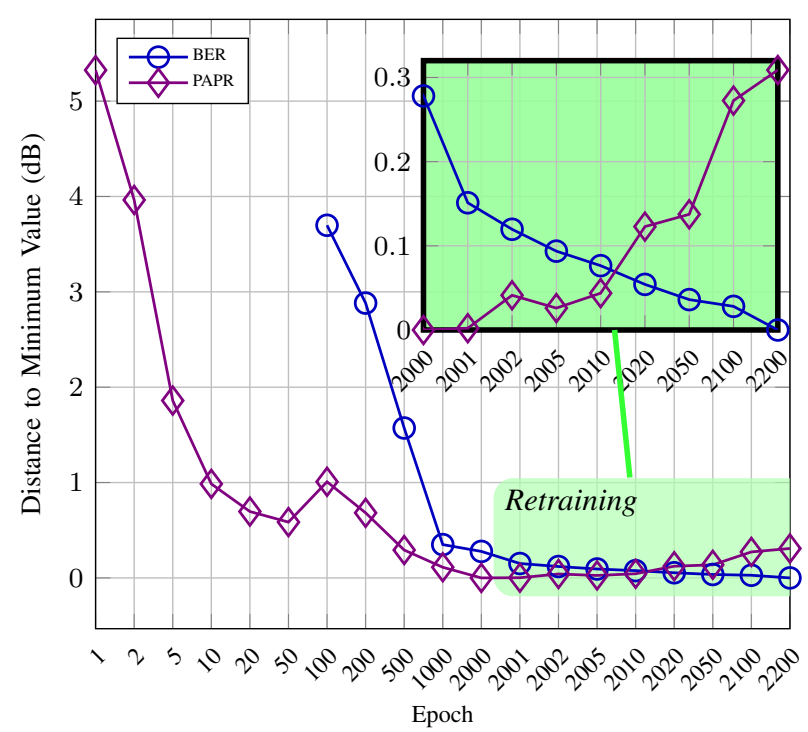

Fig. 11. Offline training complexity versus BER and PAPR improvement for IMDD-OFDMNet. The BER curve is the $E_{b} / N_{0}$ gain for reaching the $10^{-3}$ BER, while the PAPR curve is the PAPR reduction for reaching the $10^{-3}$ PAPR CCDF.

RMAs. Since each layer is detected sequentially, the IFFT/FFT sources can be reused consecutively for the detection of consecutive layers. The RX FFT complexity of IMDD-OFDMNet may be deemed to be similar to that of ACO-OFDM, since it also contains a single FFT.

As for the complexity of the NNs, this is directly related to the number of nodes in the hidden layers. The TX depicted in Fig. 4 has only a single hidden layer with $N_{2}$ neurons, yielding a total number of $N_{1} N_{2}+N_{2} N_{3}$ real multiplications and $\left(N_{1}-\right.$ 1) $N_{2}+\left(N_{2}-1\right) N_{3}$ real additions, which jointly result in approximately $2\left(N_{1} N_{2}+N_{2} N_{3}\right)$ RMAs in total. Similarly, the RX NNs of Fig. 4 would require approximately $2\left(K N_{5,1}+\right.$ $\left.N_{5,1} N_{5,2}+N_{5,2} N_{5,3}+N_{5,3} N_{1}\right)$ RMAs. Since similar NN structures have been employed for both LACONet and IMDDOFDMNet, it is reasonable to assume that they have the same AE complexity. It is also worth scrutinizing the relationship between the number of neurons and the structure of IFFT/FFT. In this context, LACONet has $N_{3}=2 K\left(1 / 2-2^{-L-1}\right)$ and $N_{4}=K$, while IMDD-OFDMNet relies on $N_{3}=2 K, N_{4}=$ $K$, according to Tabs. II and IV.

The above analysis has been summarized in Tab. V, while a graphical comparison is provided in Fig. 12. Explicitly, Fig. 12a compares the IFFT/FFT related complexities of ACO-OFDM, LACO-OFDM, the proposed LACONet and also IMDD-OFDMNet. By contrast, Fig. 12b portrays the learningrelated complexities of LACONet and IMDD-OFDMNet. While the schemes operating with and without learning tend to impose the same IFFT/FFT complexities, it is worth noting the differences in the y-axis scales in the two figures, which indicate that the learning-related complexities tend to be higher than the IFFT/FFT-related complexities. Despite the fact that the schemes of Figs. 1 and 2, which do not involve learning, contain some additional low-complexity components, such as demapping and re-mapping, it may still be deemed fair to say that the proposed schemes relying on learning tend to improve

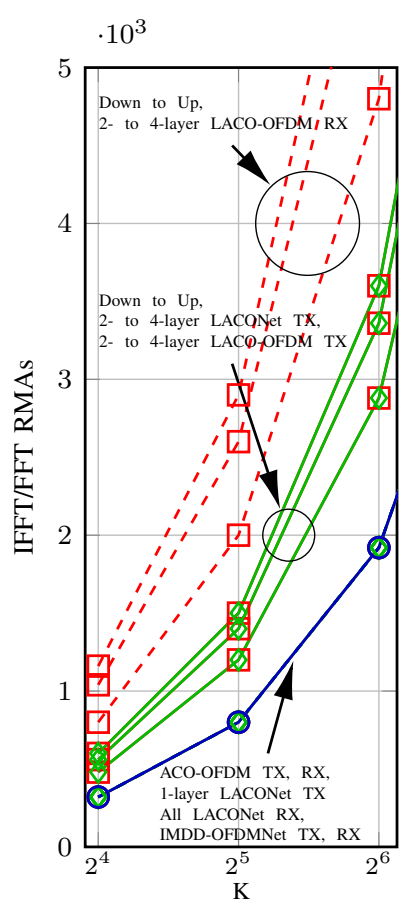

(a) IFFT/FFT-related Complexity

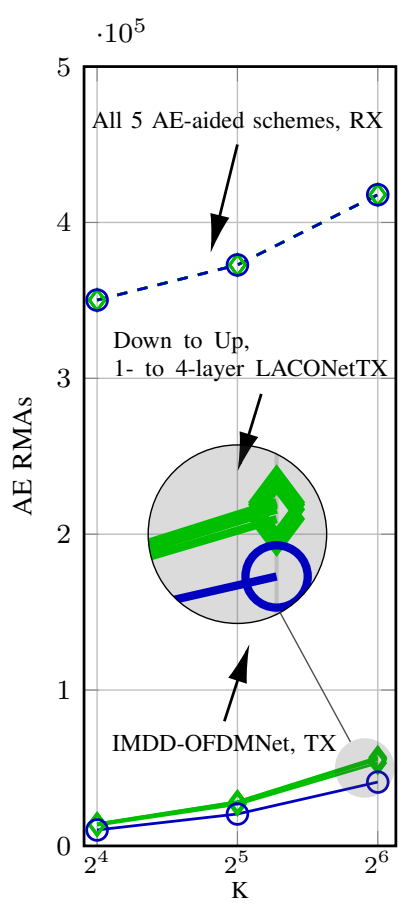

(b) Learning-Related Complexity
Fig. 12. Online processing complexity comparison for O-OFDM schemes with and without learning.

the BER and PAPR performance at the cost of a higher number of RMAs. In a nutshell, our learning-aided solutions may be deemed to dissipate more signal processing energy than their Shannonian block-based counterparts - despite the simpler architecture of Fig. 7.

\section{CONCLUSIONS}

In this paper, we proposed a pair of novel optical OFDM schemes, namely LACONet and IMDD-OFDMNet by introducing deep learning into the classic OFDM-aided architecture. Both schemes have attained better BER and PAPR performance than their conventional O-OFDM counterparts operating without learning, such as ACO-OFDM and LACOOFDM. Moreover, IMDD-OFDMNet has been shown to outperform LACONet in terms of both its BER and PAPR performance at the cost of a higher RMAs, despite its simpler architecture. More explicitly, the complexity of both the offline training and of the on-line processing of NNs, has proved to be considerably higher than that of the classic Shannonian systems adopting traditional QAM mapping. Nevertheless, NNs lend themselves to convenient parallelization, hence these complexity considerations require further research.

However, having discussed the various benefits of the AIaided solutions, the scalability limitation of the neural networks remains an impediment. As a remedy, in our future work we plan to employ a model-based solution to deal with this. Explicitly, in our future work, we will split the input stream into several groups, where each group processes the autoencoder operation simultaneously but independently. The reduced input size of the individual groups will result in a more manageable neural network size, so that the overall number 
TABLE V

ONLINE PROCESSING COMPLEXITY OF O-OFDM SCHEMES WITH AND WITHOUT LEARNING

\begin{tabular}{cllll}
\hline \multirow{2}{*}{ Scheme } & \multicolumn{2}{c}{ Transmitter (TX) } & Receiver (RX) \\
\cline { 2 - 5 } & IFFT/FFT & AE & IFFT/FFT & AE \\
\hline ACO-OFDM & $C_{1}$ & N/A & $C_{1}$ & N/A \\
LACO-OFDM & $\left(2-2^{1-L}\right) C_{1}$ & N/A & $\left(4-2^{1-L}-2^{2-L}\right) 2 C_{1}$ & N/A \\
LACONet & $\left(2-2^{1-L}\right) C_{1}$ & $2\left(N_{1} N_{2}+N_{2} N_{3}\right)$ & $C_{1}$ & $2\left(K N_{5,1}+N_{5,1} N_{5,2}+N_{5,2} N_{5,3}+N_{5,3} N_{1}\right)$ \\
IMDD-OFDMNet & $C_{1}$ & $2\left(N_{1} N_{2}+N_{2} N_{3}\right)$ & $C_{1}$ & $2\left(K N_{5,1}+N_{5,1} N_{5,2}+N_{5,2} N_{5,3}+N_{5,3} N_{1}\right)$ \\
\hline
\end{tabular}

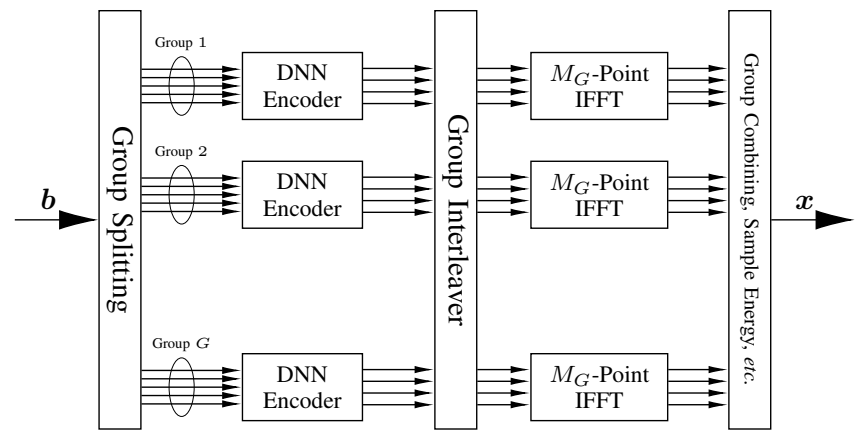

Fig. 13. Schematic of a multi-group IMDD-OFDMNet transmitter.

of subcarriers can be increased. Fig. 13 shows the tentative transmitter architecture of the proposed multi-group scheme, where the input is divided into $G$ sub-streams for individual DNN encoding and OFDM IFFT operations, before combining them for further processing. Moreover, an interleaver is placed across all groups between the DNN encoder and IFFT stage for mitigating the potential PAPR imposed by the distributed DNN training.

\section{REFERENCES}

[1] L. Hanzo, H. Haas, S. Imre, D. O'Brien, M. Rupp, and L. Gyongyosi, "Wireless myths, realities, and futures: From $3 \mathrm{G} / 4 \mathrm{G}$ to optical and quantum wireless," Proc. IEEE, vol. 100, no. Special Centennial Issue, pp. 1853-1888, May 2012.

[2] Z. Wang, Q. Wang, W. Huang, and Z. Xu, Visible light communications: Modulation and signal processing. Hoboken, NJ, USA: Wiley, 2018.

[3] J. Armstrong, "OFDM for optical communications," J. Lightw. Technol., vol. 27, no. 3, pp. 189-204, Feb. 2009.

[4] L. Hanzo, M. Münster, B. Choi, and T. Keller, OFDM and MC-CDMA for broadband multi-user communications, WLANs and broadcasting. Chichester, U.K.: Wiley, 2005.

[5] L. Hanzo, Y. Akhtman, J. Akhtman, L. Wang, and M. Jiang, MIMOOFDM for LTE, WiFi and WiMAX: Coherent versus non-coherent and cooperative turbo transceivers. Chichester, U.K.: Wiley, 2011.

[6] J. Armstrong and A. J. Lowery, "Power efficient optical OFDM," Electron. Lett., vol. 42, no. 6, Mar. 2006.

[7] R. Mesleh, H. Elgala, and H. Haas, "On the performance of different OFDM based optical wireless communication systems," IEEE J. Opt. Commun. Netw., vol. 3, no. 8, pp. 620-628, Aug. 2011.

[8] R. Zhang and L. Hanzo, "Multi-layer modulation for intensity-modulated direct-detection optical OFDM," IEEE J. Opt. Commun. Netw., vol. 5, no. 12, pp. 1402-1412, Dec. 2013.

[9] Q. Wang, Z. Wang, and L. Dai, "Asymmetrical hybrid optical ofdm for visible light communications with dimming control," IEEE Photon. Technol. Lett., vol. 27, no. 9, pp. 974-977, May 2015.

[10] X. Zhang, Q. Wang, R. Zhang, S. Chen, and L. Hanzo, "Performance analysis of layered ACO-OFDM," IEEE Access, vol. 5, pp. 18366 18381, 2017.

[11] J. Zhou and W. Zhang, "A comparative study of unipolar OFDM schemes in Gaussian optical intensity channel," IEEE Trans. Commun., vol. 66, no. 4, pp. 1549-1564, Apr. 2018.
[12] X. Zhang, Z. Babar, P. Petropoulos, H. Haas, and L. Hanzo, "The evolution of optical OFDM," IEEE Commun. Surveys Tuts., vol. 23, no. 3, pp. 1430-1457, 3rd Quart. 2021.

[13] X. Zhang, Z. Babar, S. Chen, and L. Hanzo, "Multi-class coded layered asymmetrically clipped optical OFDM," IEEE Trans. Commun., vol. 67, no. 1, pp. 578-589, Jan. 2019.

[14] X. Zhang, S. Chen, and L. Hanzo, "On the discrete-input continuousoutput memoryless channel capacity of layered ACO-OFDM," J. Lightw. Technol., vol. 38, no. 18, pp. 4955-4968, Sep. 2020.

[15] T. O'Shea and J. Hoydis, "An introduction to deep learning for the physical layer," IEEE Trans. on Cogn. Commun. Netw., vol. 3, no. 4, pp. 563-575, Apr. 2017.

[16] I. Goodfellow, Y. Bengio, A. Courville, and Y. Bengio, Deep learning. MIT press Cambridge, 2016, vol. 1.

[17] J. Wang, C. Jiang, H. Zhang, Y. Ren, K.-C. Chen, and L. Hanzo, "Thirty years of machine learning: The road to Pareto-optimal wireless networks," IEEE Commun. Surveys Tuts., vol. 22, no. 3, pp. 1472-1514, 3rd Quart. 2020.

[18] A. Fehske, J. Gaeddert, and J. H. Reed, "A new approach to signal classification using spectral correlation and neural networks," in $1 s t$ IEEE Intl. Symposium on New Frontiers in Dynamic Spectrum Access Networks, 2005. DySPAN 2005. Baltimore, MD, USA: IEEE, Nov. 2005, pp. 144-150.

[19] S. Cammerer, F. A. Aoudia, S. Dörner, M. Stark, J. Hoydis, and S. ten Brink, "Trainable communication systems: Concepts and prototype," IEEE Trans. Commun., vol. 68, no. 9, pp. 5489-5503, Sep. 2020.

[20] H. Ye, G. Y. Li, and B.-H. Juang, "Power of deep learning for channel estimation and signal detection in OFDM systems," IEEE Wireless Commun. Lett., vol. 7, no. 1, pp. 114-117, Feb. 2018.

[21] Y. Jin, J. Zhang, S. Jin, and B. Ai, "Channel estimation for cell-free mmWave massive MIMO through deep learning," IEEE Trans. Veh. Technol., vol. 68, no. 10, pp. 10325-10329, Oct. 2019.

[22] T. V. Luong, Y. Ko, N. A. Vien, D. H. N. Nguyen, and M. Matthaiou, "Deep learning-based detector for OFDM-IM," IEEE Wireless Commun. Lett., vol. 8, no. 4, pp. 1159-1162, Aug. 2019.

[23] J. Wang, C. Jiang, H. Zhang, X. Zhang, V. C. Leung, and L. Hanzo, "Learning-aided network association for hybrid indoor LiFi-WiFi systems," IEEE Trans. Veh. Technol., vol. 67, no. 4, pp. 3561-3574, Apr. 2017.

[24] N. Yang, H. Zhang, K. Long, H.-Y. Hsieh, and J. Liu, "Deep neural network for resource management in NOMA networks," IEEE Trans. Veh. Technol., vol. 69, no. 1, pp. 876-886, Jan. 2019.

[25] B. Karanov, M. Chagnon, F. Thouin, T. A. Eriksson, H. Bülow, D. Lavery, P. Bayvel, and L. Schmalen, "End-to-end deep learning of optical fiber communications," J. Lightw. Technol., vol. 36, no. 20, pp. 48434855, Oct. 2018.

[26] Z.-R. Zhu, J. Zhang, R.-H. Chen, and H.-Y. Yu, "Autoencoder-based transceiver design for OWC systems in log-normal fading Channel," IEEE Photon. J., vol. 11, no. 5, pp. 1-12, Oct. 2019.

[27] A. Felix, S. Cammerer, S. Dörner, J. Hoydis, and S. Ten Brink, "OFDMautoencoder for end-to-end learning of communications systems," in IEEE SPAWC, 2018, pp. 1-5.

[28] T. V. Luong, Y. Ko, N. A. Vien, M. Matthaiou, and H. Q. Ngo, "Deep energy autoencoder for noncoherent multicarrier MU-SIMO systems," IEEE Trans. Wireless Commun., vol. 19, no. 6, pp. 3952-3962, 2020.

[29] B. Karanov, D. Lavery, P. Bayvel, and L. Schmalen, "End-to-end optimized transmission over dispersive intensity-modulated channels using bidirectional recurrent neural networks," Opt. Exp., vol. 27, no. 14, pp. 19650-19663, 2019.

[30] L. Hao, D. Wang, W. Cheng, J. Li, and A. Ma, "Performance enhancement of ACO-OFDM-based VLC systems using a hybrid autoencoder scheme," Opt. Commun., vol. 442, pp. 110-116, Jul. 2019. 
[31] M. G. Ulkar, T. Baykas, and A. E. Pusane, "VLCnet: Deep learning based end-to-end visible light communication system," J. Lightw. Technol., 2020.

[32] C. Chen, D. Basnayaka, and H. Haas, "Non-line-of-sight channel impulse response characterisation in visible light communications," in 2016 IEEE Intl. Conf. Commun. (ICC). Kuala Lumpur, Malaysia: IEEE, May 2016, pp. 1-6.

[33] K. Lee, H. Park, and J. R. Barry, "Indoor channel characteristics for visible light communications," IEEE Commun. Lett., vol. 15, no. 2, pp. 217-219, Feb. 2011

[34] S. D. Dissanayake and J. Armstrong, "Comparison of ACO-OFDM, DCO-OFDM and ADO-OFDM in IM/DD systems," J. Lightw. Technol., vol. 31, no. 7, pp. 1063-1072, Apr. 2013.

[35] B. Ranjha and M. Kavehrad, "Hybrid asymmetrically clipped OFDMbased IM/DD optical wireless system," IEEE J. Opt. Commun. Netw., vol. 6, no. 4, pp. 387-396, Apr. 2014.

[36] N. Wu and Y. Bar-Ness, "A novel power-efficient scheme asymmetrically and symmetrically clipping optical (ASCO)-OFDM for IM/DD optical systems," EURASIP J. Adv. Signal Process., vol. 2015, no. 3, pp. 1-10, Jan. 2015.

[37] R. Bai, Q. Wang, and Z. Wang, "Asymmetrically clipped absolute value optical OFDM for intensity-modulated direct-detection systems," J. Lightw. Technol., vol. 35, no. 17, pp. 3680-3691, Sep. 2017.

[38] S. Ioffe and C. Szegedy, "Batch normalization: Accelerating deep network training by reducing internal covariate shift," in Proc. 32nd Intl. Conf. Mach. Learn. Lille, France: PMLR, Jul. 2015, pp. 448-456.

[39] L. Hanzo, T. H. Liew, B. L. Yeap, R. Y. S. Tee, and S. X. Ng, Turbo coding, turbo equalisation and space-time coding: EXIT-chart-aided near-capacity designs for wireless channels, 2 nd ed. Chichester: WileyIEEE Press, 2011.

Xiaoyu Zhang (Member, IEEE) received the B.Eng. degree in electronic information engineering from the University of Electronic Science and Technology of China (UESTC), Chengdu, China, in 2014, and the M.Sc. and Ph.D. degrees from the University of Southampton, Southampton, U.K., in 2015 and 2020, respectively. He is currently a Research Fellow in Optical Wireless Communications at the University of Southampton, where he is affiliated with both the Next Generation Wireless Group and the Optoelectronics Research Centre. His research interests include the modulation, signal processing, performance analysis and optimization for wireless and optical communications.

Thien Van Luong is currently a Lecturer with the Faculty of Computer Science, Phenikaa University, Vietnam. He was a Research Fellow with University of Southampton, U.K. Prior to that he obtained the Ph.D. degree at Queen's University Belfast, U.K., and the B.S. degree at Hanoi University of Science and Technology, Vietnam. His research interests include applied machine learning in signal processing and wireless communications.

Periklis Petropoulos (Fellow, OSA) graduated from the Department of Electrical Engineering and Information Technology, University of Patras. He received an MSc degree in Communications Engineering from the University of Manchester Institute of Science and Technology and a $\mathrm{PhD}$ degree in Optical Telecommunications from the Optoelectronics Research Centre (ORC), University of Southampton, where he currently works as a Professor. His research interests lie in the fields of optical communications, all-optical signal processing and novel fibre and waveguide technologies and he leads national research projects in these areas. His research has been published extensively in technical journals and conference proceedings, including several invited and post-deadline papers at major international conferences.

Prof Petropoulos is the Editor-in-Chief of the IET Optoelectronics technical journal. He has served both as member and sub-committee chair of the Technical Programme Committees for several international conferences, including the European Conference on Optical Communication (ECOC) and the Optical Fiber Communication (OFC) conference. He is a Fellow of the Optical Society of America.
Lajos Hanzo (Fellow, IEEE) (http://www-mobile.ecs.soton.ac.uk/, https://en.wikipedia.org/wiki/Lajos_Hanzo) received his Master degree and Doctorate in 1976 and 1983, respectively from the Technical University (TU) of Budapest. He was also awarded the Doctor of Sciences (DSc) degree by the University of Southampton (2004) and Honorary Doctorates by the TU of Budapest (2009) and by the University of Edinburgh (2015). He is a Foreign Member of the Hungarian Academy of Sciences and a former Editor-in-Chief of the IEEE Press. He has served several terms as Governor of both IEEE ComSoc and of VTS. He has published 1900+ contributions at IEEE Xplore, 19 Wiley-IEEE Press books and has helped the fast-track career of $123 \mathrm{PhD}$ students. Over 40 of them are Professors at various stages of their careers in academia and many of them are leading scientists in the wireless industry. He is also a Fellow of the Royal Academy of Engineering (FREng), of the IET and of EURASIP. 